\title{
An Investigation Comparing the Efficacy of Topical Ocular Application of Tacrolimus and Cyclosporine in Dogs
}

\author{
Diane V .H. Hendrix, ${ }^{1}$ Elizabeth A. Adkins, ${ }^{2}$ Daniel A. Ward, ${ }^{1}$ Jamie Stuffle, ${ }^{1}$ \\ and Brian Skorobohach ${ }^{3}$ \\ ${ }^{1}$ Department of Small Animal Clinical Sciences, College of Veterinary Medicine, The University of Tennessee, \\ 2407 River Drive, Knoxville, TN 37996, USA \\ ${ }^{2}$ The Hope Center for Advanced Veterinary Medicine, Vienna, VA 22180, USA \\ ${ }^{3}$ Calgary Animal Referral and Emergency Centre, 12th Street Southeast, Calgary, AB, Canada T2H 2Y4
}

Correspondence should be addressed to Diane V.H. Hendrix, dhendrix@utk.edu

Received 22 January 2011; Accepted 29 March 2011

Academic Editor: Michael Stoskopf

Copyright ( 2011 Diane V .H. Hendrix et al. This is an open access article distributed under the Creative Commons Attribution License, which permits unrestricted use, distribution, and reproduction in any medium, provided the original work is properly cited.

\begin{abstract}
The purpose of this paper was to determine the efficacy and safety of topical tacrolimus, compared to cyclosporine, for treating keratoconjunctivitis sicca (KCS) in dogs. This study was a two-phase, randomized, controlled, masked clinical trial. Phase 1 evaluated ophthalmic $0.03 \%$ tacrolimus in normal dogs. Ocular examinations were performed daily. Phase 2 evaluated the efficacy of tacrolimus in treating KCS. Half the dogs received $2 \%$ cyclosporine A; the others received $0.03 \%$ tacrolimus, both diluted in olive oil. Four ophthalmic examinations were done over 12 weeks. There was no significant difference between groups in phase I. In phase 2, there was no significant difference in Schirmer tear test I (STT) results between the two groups, and both groups had a significant increase in STT over time. Both drugs were effective in increasing the STT in dogs naïve to lacrimostimulants. Tacrolimus was effective in increasing the STT in 4 dogs currently nonresponsive to cyclosporine.
\end{abstract}

\section{Introduction}

Keratoconjunctivitis sicca (KCS) is a deficiency of the aqueous layer of the precorneal tear film. Clinical signs in dogs with KCS include mucopurulent ocular discharge, conjunctivitis, keratitis, and blepharospasm. Dogs with KCS have a Schirmer tear test I (STT) result of $<10 \mathrm{~mm} /$ minute, and severely affected dogs often have an STT result of $0 \mathrm{~mm} / \mathrm{min}$. While many causes of KCS in dogs have been described [1-10], KCS in dogs is most often considered to be immune-mediated based on studies that showed increased numbers of lymphocytes and plasma cells with acinar atrophy in the lacrimal glands [11, 12]. Immunemediated KCS is typically bilateral and affects many breeds.

Treatment of immune-mediated KCS is aimed at decreasing the immune response in the lacrimal gland and restoring aqueous tear production. Cyclosporine A (CsA) is a commonly used and effective treatment for canine KCS [1316]. Formulations typically used are previously commercially available as $0.2 \%$ CsA ophthalmic ointment (Optimmune), and $1 \%$ and $2 \%$ CsA in olive or corn oil [13-19]. In addition to increasing tear production by inhibiting T-helper lymphocyte proliferation and infiltration of lacrimal gland acini, CsA restores conjunctival goblet cell mucin production $[12,13,17,18]$. Previous studies have shown that dogs treated with ophthalmic CsA treatment have a decreased lymphocyte stimulation index compared to control dogs and that there are measurable levels of CsA in peripheral blood of treated dogs $[20,21]$. However, a more recent study did not show a change in the lymphocyte stimulation index or significant blood levels with use of topical cyclosporine [22]. Unfortunately, approximately $25 \%$ of dogs with KCS do not respond to treatment with $1 \%$ or $2 \%$ CsA [15-17].

Tacrolimus is a newer immunosuppressant agent that has been used for the treatment of immune-mediated KCS in dogs [23]. Tacrolimus is a macrolide antibiotic that has a similar immunopharmacological profile as CsA but is approximately 100-times more potent than CsA [24-26]. In 2005, the United Stated Federal Drug Administration issued an advisory for the potential carcinogenic effects of 
the dermal tacrolimus when used on humans. This advisory recommended that tacrolimus only be used as a secondline agent for short-term and intermittent use [27]. This advisory was based on a small number of patients, and the ramifications for dogs being treated with tacrolimus for KCS are not known.

Both CsA and tacrolimus are calcineurin inhibitors that reversibly inhibit T-cell proliferation and prevent the release of proinflammatory cytokines. Calcineurin inhibitors bind to intracellular immunophilins and form complexes that subsequently bind to and inhibit calcineurin. In blocking calcineurin, translocation of the cytoplasmic component of the nuclear factor of activated $\mathrm{T}$ cells to the nucleus is prevented. This prevention of translocation impairs transcription of the genes encoding IL-2 and other cytokines, thereby suppressing T-cell proliferation and normal immune function [28].

The first purpose of this study was to determine the safety of topical tacrolimus in normal dogs. The second purpose was to compare the efficacy of topical ophthalmic application of $0.03 \%$ tacrolimus with that of $2 \%$ CsA in olive oil in a double-masked study.

\section{Materials and Methods}

This study was designed as a two-phase, controlled, masked, randomized clinical trial. Phase 1 was a toxicity study, and phase 2 was an efficacy study. This study was approved by the University of Tennessee Animal Care and Use Committee. Thirteen clinically normal research beagles were treated in the toxicity study. Prior to inclusion in the study, all dogs received complete ophthalmic examinations including STT, fluorescein staining, tonometry, slit-lamp biomicroscopy, and indirect ophthalmoscopy. Each dog was randomly assigned to either the tacrolimus or control group. Seven dogs were treated twice daily topically with the injectable form of tacrolimus ( $5 \mathrm{mg} / \mathrm{mL}$; Fujisawa Inc., Japan) formulated into a $0.03 \%$ concentration in an olive oil vehicle. Six dogs were treated twice daily with the olive oil vehicle alone. All dogs were treated for 14 days. All dogs received daily ophthalmic examinations including STT, fluorescein staining, and slit lamp biomicroscopy during the treatment period. Daily clinical scores were assigned to each dog grading the severity of conjunctival hyperemia, blepharospasm, and epiphora by a masked observer (EA). The grading scale consisted of grades 0 through 3 for each of the criteria evaluated. Grade 0 was considered normal, grade 1 mildly affected, grade 2 moderately affected, and grade 3 severely affected. Serum biochemistry profiles were evaluated on days 0 and 15 to determine if the treatment caused any alterations in these values.

For the phase 2 efficacy study, patients were enrolled for 12 weeks. Inclusion criteria consisted of an STT result less than $10 \mathrm{~mm} / \mathrm{minute}$ in both eyes (OU) even with current CsA treatment, clinical signs of KCS OU, and written consent of the owner. Exclusion criteria included corneal ulceration, suspected bacterial keratitis, systemic neoplasia, immunosuppressive therapy for a systemic disease, and previous treatment with sulfonamides or etodolac.
Dogs were randomly assigned to 1 of 2 groups. One group was treated with $2 \% \mathrm{CsA}$ in olive oil OU BID, and the other group was treated with $0.03 \%$ tacrolimus in olive oil OU BID. Any medications that the patient was currently receiving to treat KCS such as antibiotic ointment and ocular lubricants were continued during the course of the study. Five dogs in the CsA group were already being treated with CsA (three with $2 \%$ solution and two with $0.2 \%$ ointment). Six dogs in the tacrolimus group were already being treated with CsA (four with $2 \%$ solution and two with $0.2 \%$ ointment). Upon enrollment, the current CsA formulation was discontinued and the CsA or tacrolimus treatment was initiated without having a washout period. Criteria used to terminate participation in the study included corneal ulceration, development of bacterial keratitis, or signs of excessive ocular irritation secondary to drug administration.

Each dog was evaluated on day 0 and at weeks 4, 8, and 12 of the study by a masked observer (EA, DH, DW, or BS). Each dog had a complete ophthalmic examination at each evaluation, including STT, fluorescein staining, slit lamp biomicroscopy, and indirect ophthalmoscopy. Serum biochemistry profiles were evaluated at weeks 0 and 4 of the study.

Both eyes of each dog in the study were photographed at each visit and were graded by a masked observer (DH). Clinical signs were evaluated as follows: conjunctival hyperemia (score 0: no conjunctival hyperemia, 1: mild hyperemia, 2: moderate hyperemia), ocular discharge (score 0: no ocular discharge, 1: slight ocular discharge at medial canthus, 2: discharge across cornea or on eyelid margins), area of corneal pigmentation (score 0: no corneal pigmentation, 1: pigment over less than $25 \%$ of the cornea, 2: pigment over $25-50 \%$ of the cornea, 3: pigment over $50-75 \%$ of the cornea, 4 : pigment over $>75 \%$ of the cornea), corneal pigment density (score 0 : no pigment, 1: iris easily visualized through the pigment, 2: iris partially visualized through the pigment, 3: iris not visible through the pigment), corneal vessel length (Score 0-no corneal vessels, 1: limbal corneal vessels, 2: vessels halfway towards the corneal axis, 3: vessels extending to axial cornea), corneal vessel density (Score 0: no vessels, 1: vessels extending to cornea from $<25 \%$ of the circumference of the limbus, 2: vessels extending to cornea from $25-50 \%$ of the circumference of the limbus, 3: vessels extending to cornea from $50-75 \%$ of the circumference of the limbus, 4 : vessels extending to cornea from $>75 \%$ of the circumference of the limbus), and corneal keratinization (score 0: none, 1: mild opacity, 2: moderate opacity).

Data from both eyes from each dog were combined for statistical analysis. For phase 1, the daily clinical scores and STT results between the two groups were compared by Student's $t$-test. Within each group the effect of treatment on STT over time was evaluated by repeated measures analysis of variance (RM-ANOVA).

For the clinical study, the data from both eyes from each dog was combined. Within each group the effect of the treatments on STT and clinical scores over time was evaluated by one-way RM-ANOVA. When normality failed, the Freidman repeated measures ANOVA on ranks was used. The STT and clinical scores comparing the CsA and tacrolimus groups on day 0 , and at weeks 4,8 , and 12 were evaluated by Student's 
$t$-test. Statistical significance was set at $P \leq .05$. Statistical analyses were done with SPSS software (SPSS Inc., Chicago, Illinois).

\section{Results}

For all dogs in phase 1, the serum biochemistries were within reference ranges on day 0 and day 15 . There was no difference between the two groups in the daily clinical scores (data not shown). Mean STT on day 0 was $19.08( \pm 2.7)$ for the control group and $20.64( \pm 3.2)$ in the tacrolimus group. On day 15 , the mean STT was $21.08( \pm 2.3)$ for the control group and $24.29( \pm 3.2)$ for the tacrolimus group. There was no statistical difference in clinical scores between groups on day 0 or day 15 or between STT on day 0 and day 15 over time in either group.

Of the 29 dogs enrolled in the efficacy study, 18 dogs completed the study, with 10 dogs in the CsA group and eight dogs in the tacrolimus group. Of the 18 dogs, 16 were pure breeds, representing 14 different breeds, and two were mixed-breed dogs. Nine were spayed females, one was an intact female, four were neutered males, and four were intact males. Mean age in the CsA group was 8 years, and mean age in the tacrolimus group was 9 years. Five dogs in the CsA group and six dogs in the tacrolimus group had been treated with CsA prior to enrolling in the study.

Six dogs in the CsA group and eight dogs in the tacrolimus group had photographic images from all visits available for scoring. There was no significant difference in combined clinical scores for conjunctival hyperemia, ocular discharge, corneal pigmentation, corneal vascularization, or keratinization over time for the CsA or tacrolimus groups. There was no statistical difference in clinical scores between the CsA group and tacrolimus group at day 0 , week 4 , or week 8. There was a significant difference in the clinical scores between the CsA group and the tacrolimus group at week $12(P=.040)$. The CsA group had lower clinical scores than the tacrolimus group at all time points, although this was not significant except at week 12.

Both groups had an increase in mean tear production over time. In both groups, the STT was higher at the fourth visit (CsA group: median $11 \mathrm{~mm} /$ minute, range 1.521.5; tacrolimus group: median $9.75 \mathrm{~mm} / \mathrm{min}$, range 7 $20 \mathrm{~mm} /$ minute) compared to the first visit (CsA group: median $5 \mathrm{~mm} /$ minute, range $0-8.5 \mathrm{~mm} /$ minute; tacrolimus group: median $4.5 \mathrm{~mm} /$ minute, range $0-6.5$ ). This difference between the STT at time 0 and at week 12 in both groups was significant (CsA, $P<.001$, tacrolimus $P=.001$; Table 1 ).

There was no significant difference in mean STT between the groups at time 0,4 weeks, 8 weeks, or 12 weeks (Table 2).

No dogs had any signs of irritation from either drug. Serum biochemistry values were within normal limits for all patients.

Four of five dogs in the CsA group not previously treated with lacrimostimulants responded with an increase in tear production $>5 \mathrm{~mm} /$ minute when treated with CsA. There was no change in the tear production in the one dog that did not respond. Of the five dogs in the CsA group that were already being treated with CsA (three with $2 \%$ solution
TABLE 1: Median tear production (STT) in $\mathrm{mm} / \mathrm{min}$ at baseline and week 12 for the cyclosporine group and the tacrolimus group.

\begin{tabular}{lccc}
\hline Group & Baseline (range) & 12 weeks (range) & $P$ value \\
\hline Cyclosporine & $5(0-8.5)$ & $11(1.5-21.5)$ & $P<.001$ \\
Tacrolimus & $4.5(0-6.5)$ & $9.75(7-20)$ & $P=.001$ \\
\hline
\end{tabular}

TABle 2: Mean tear production (STT) in $\mathrm{mm} / \mathrm{min}$ at baseline, 4 weeks, 8 weeks, and 12 weeks for the cyclosporine group and the tacrolimus group. There was no difference between groups at any time.

\begin{tabular}{lccc}
\hline Time (weeks) & Cyclosporine & Tacrolimus & $P$ value \\
\hline Baseline & 4.6 & 3.2 & $P=.336$ \\
4 & 9.3 & 7.0 & $P=.302$ \\
8 & 10.8 & 10.0 & $P=.768$ \\
12 & 11.7 & 11.1 & $P=.850$ \\
\hline
\end{tabular}

and two with $0.2 \%$ ointment), only one dog, which was being treated with $2 \%$ CsA prior to being enrolled in the study, had an increase in STT of $>5 \mathrm{~mm} /$ minute. Only two dogs in the tacrolimus group were not already being treated with CSA at time of enrollment in the study, and they both responded with a tear production increase $>5 \mathrm{~mm} /$ minute. Four of the six dogs in the tacrolimus group that were already being treated with CsA (two with $2 \%$ CsA in oil and two with $0.2 \%$ CsA ointment) had an increase in mean STT $>5 \mathrm{~mm} /$ minute. Two dogs did not have a mean increase in STT $>5 \mathrm{~mm} /$ minute. One of those had a tear production of $5 \mathrm{~mm} / \mathrm{minute}$ in the right eye with no change during the study and the left eye increased from $8 \mathrm{~mm} /$ minute in to $12 \mathrm{~mm} /$ minute at 12 weeks. The other dog was similar and had no change in the right eye, but the STT increased from $0 \mathrm{~mm} /$ minute in the left eye upon enrollment to $9 \mathrm{~mm} /$ minute at 12 weeks.

\section{Discussion}

The results of this small, short-term study support tacrolimus ophthalmic solution as potentially successful in increasing tear production in dogs nonresponsive to treatment with CsA. As previously reported, both CsA and tacrolimus are useful in the treatment of dogs with KCS previously not treated with lacrimostimulants [13-16, 23].

A previous study investigating the effects of $2 \%$ CsA in olive oil administered TID in normal beagles showed a significant increase in STT after 7 days. Our study did not show a significant increase in tear production between the dogs treated with $0.03 \%$ tacrolimus in olive oil and control dogs treated with the olive oil alone after 14 days of BID treatment. However, a direct comparison between the two studies cannot be made because of the difference in treatment frequency.

While the numbers are small, having a response of $>5 \mathrm{~mm} /$ minute STT in the majority of dogs naïve to CsA was expected, as was having most of the dogs that were already 
being treated with CsA not show a response. The single dog in the CsA group that was already being treated with $2 \%$ CsA that responded to the study CsA with an increase in STT $>5 \mathrm{~mm} /$ minute may have done so simply because of increased owner compliance. Of the eight dogs in the tacrolimus group, two that were naïve to lacrimostimulant therapy and four that were already being treated with CsA (two with $2 \%$ CsA in oil and two with $0.2 \%$ CsA ointment) responded to the tacrolimus. While the numbers are small in this study (using tacrolimus as a $0.03 \%$ solution in olive oil), these results support the findings by Berdoulay who used $0.02 \%$ aqueous solution that tacrolimus may be effective at increasing tear production in dogs nonresponsive to CsA [23].

Significant improvement in clinical signs over time was not seen in this study. This is in contrast to previous studies evaluating CsA and tacrolimus that found a decrease in severity of signs both with and without increased tear production $[17,23]$. In contrast to those studies, which evaluated only ocular discharge and signs of conjunctivitis, the current study also assessed corneal pigmentation, corneal vascularization, and corneal keratinization. These signs develop with chronicity and are also expected to require more time to improve. The clinical signs of the dogs of this study may have improved significantly with a longer followup period.

This study used olive oil rather than a lactose cellulose base for the increased lubrication and contact time associated with an oil base. Similar efficacy of the tacrolimus in olive oil compared to the aqueous base was seen, and there were no complications seen with the olive oil base [23]. Therefore, it may be the formulation of choice in dogs with very low tear production or in dogs with increased corneal exposure secondary to lagophthalmos.

CsA was used as the control in this study to ensure that no eyes deteriorated due to lack of standard and proven treatment of KCS. To assure that differences between the two drugs could not be attributed to different vehicles, both the CsA and tacrolimus were diluted in olive oil. Unfortunately for this study, most dogs in our region are treated appropriately for KCS, and, therefore, we were not able to obtain large numbers of dogs that had not had CsA therapy initiated prior to the study.

\section{Acknowledgments}

This paper was supported by the American College of Veterinary Ophthalmologists, University of Tennessee Department of Small Animal Clinical Sciences, University of Tennessee Center of Excellence in Livestock Diseases and Human Health, and the Rasmussen Charitable Gift Fund. The authors would like to thank Misty Bailey for critical review of the paper.

\section{References}

[1] S. L. Berger, R. H. Scagliotti, and E. M. Lund, "A quantitative study of the effects of tribrissen on canine tear production," Journal of the American Animal Hospital Association, vol. 31, no. 3, pp. 236-241, 1995.
[2] R. V. Morgan and A. J. Bachrach, "Keratoconjunctivitis sicca associated with sulfonamide therapy in dogs," Journal of the American Veterinary Medical Association, vol. 180, no. 4, pp. 432-434, 1982.

[3] G. Klauss, E. A. Giuliano, C. P. Moore et al., "Keratoconjunctivitis sicca associated with administration of etodolac in dogs: 211 cases (1992-2002)," Journal of the American Veterinary Medical Association, vol. 230, no. 4, pp. 541-547, 2007.

[4] S. R. Hollingsworth, D. D. Canton, N. C. Buyukmihci, and T. B. Farver, "Effect of topically administered atropine on tear production in dogs," Journal of the American Veterinary Medical Association, vol. 200, no. 10, pp. 1481-1484, 1992.

[5] I. P. Herring, J. P. Pickett, E. S. Champagne, and M. Marini, "Evaluation of aqueous tear production in dogs following general anesthesia," Journal of the American Animal Hospital Association, vol. 36, no. 5, pp. 427-430, 2000.

[6] T. J. Kern and H. N. Erb, "Facial neuropathy in dogs and cats: 95 cases (1975-1985)," Journal of the American Veterinary Medical Association, vol. 191, no. 12, pp. 1604-1609, 1987.

[7] R. Carter and C. M. H. Colitz, "The causes, diagnosis, and treatment of canine keratoconjunctivitis sicca," Veterinary Medicine, vol. 97, no. 9, pp. 683-694, 2002.

[8] B. H. Grahn and L. S. Sandmeyer, "Diseases and surgery of the canine nasolacrimal system," in Veterinary Ophthalmology, K. N. Gelatt, Ed., pp. 618-661, Blackwell Publishing, 2007.

[9] R. V. Morgan, J. M. Duddy, and K. Mcclurg, "Prolapse of the gland of the third eyelid in dogs: a retrospective study of 89 cases (1980-1990)," Journal of the American Animal Hospital Association, vol. 29, no. 1, pp. 56-60, 1993.

[10] H. D. Westermeyer, D. A. Ward, and K. Abrams, "Breed predisposition to congenital alacrima in dogs," Veterinary Ophthalmology, vol. 12, no. 1, pp. 1-5, 2009.

[11] R. L. Kaswan, C. L. Martin, and W. L. J. Chapman, "Keratoconjunctivitis sicca: histopathologic study of nictitating membrane and lacrimal glands from 28 dogs," American Journal of Veterinary Research, vol. 45, no. 1, pp. 112-118, 1984.

[12] D. I. Bounous, K. P. Carmichael, R. L. Kaswan, S. Hirsh, and J. Stiles, "Effects of ophthalmic cyclosporine on lacrimal gland pathology and function in dogs with keratoconjunctivitis sicca," Veterinary and Comparative Ophthalmology, vol. 5, no. 1, pp. 5-12, 1995.

[13] R. L. Kaswan, M. A. Salisbury, and D. A. Ward, "Spontaneous canine keratoconjunctivitis sicca: a useful model for human keratoconjunctivitis sicca: treatment with cyclosporine eye drops," Archives of Ophthalmology, vol. 107, no. 8, pp. 1210-1216, 1989.

[14] M. A. Salisbury, R. L. Kaswan, D. A. Ward, C. L. Martin, J. M. Ramsey, and C. A. Fischer, "Topical application of cyclosporine in the management of keratoconjunctivitis sicca in dogs," Journal of the American Animal Hospital Association, vol. 26, no. 3, pp. 269-274, 1990.

[15] D. K. Olivero, M. G. Davidson, R. V. English, M. P. Nasisse, V. E. Jamieson, and T. M. Gerig, "Clinical evaluation of $1 \%$ cyclosporine for topical treatment of keratoconjunctivitis sicca in dogs," Journal of the American Veterinary Medical Association, vol. 199, no. 8, pp. 1039-1042, 1991.

[16] R. V. Morgan and K. L. Abrams, "Topical administration of cyclosporine for treatment of keratoconjunctivitis sicca in dogs," Journal of the American Veterinary Medical Association, vol. 199, no. 8, pp. 1043-1046, 1991.

[17] C. P. Moore, J. B. Mchugh, J. G. Thorne, and T. E. Phillips, "Effect of cyclosporine on conjunctival mucin in a canine keratoconjunctivitis sicca model," Investigative Ophthalmology and Visual Science, vol. 42, no. 3, pp. 653-659, 2001. 
[18] C. Izci, I. Celik, F. Alkan et al., "Histologic characteristics and local cellular immunity of the gland of the third eyelid after topical ophthalmic administration of $2 \%$ cyclosporine for treatment of dogs with keratoconjunctivitis sicca," American Journal of Veterinary Research, vol. 63, no. 5, pp. 688-694, 2002.

[19] R. Ofri, G. N. Lambrou, I. Allgoewer et al., "Clinical evaluation of pimecrolimus eye drops for treatment of canine keratoconjunctivitis sicca: a comparison with cyclosporine a," Veterinary Journal, vol. 179, no. 1, pp. 70-77, 2009.

[20] B. C. Gilger, J. Andrews, D. A. Wilkie et al., "Cellular immunity in dogs with keratoconjunctivitis sicca before and after treatment with topical 2\% cyclosporine," Veterinary Immunology and Immunopathology, vol. 49, no. 3, pp. 199-208, 1995.

[21] B. C. Gilger, J. Andrews, D. A, Wilkie et al., "Lymphocyte proliferation and blood drug levels in dogs with keratoconjunctivitis sicca receiving long-term topical ocular cyclosporine," and Comparative Ophthalmology, vol. 6, no. 2, pp. 125-130, 1996.

[22] D. L. Williams, "Lack of effects on lymphocyte function from chronic topical ocular cyclosporine medication: a prospective study," Veterinary Ophthalmology, vol. 13, no. 5, pp. 315-320, 2010.

[23] A. Berdoulay, R. V. English, and B. Nadelstein, "Effect of topical $0.02 \%$ tacrolimus aqueous suspension on tear production in dogs with keratoconjunctivitis sicca," Veterinary Ophthalmology, vol. 8, no. 4, pp. 225-232, 2005.

[24] E. Anglade, R. Yatscoff, R. Foster, and U. Grau, "Nextgeneration calcineurin inhibitors for ophthalmic indications," Expert Opinion on Investigational Drugs, vol. 16, no. 10, pp. 1525-1540, 2007.

[25] U. Benelli, A. Lepri, M. Del Tacca, and M. Nardi, "FK506 delays corneal graft rejection in a model of corneal xenotransplantation," Journal of Ocular Pharmacology and Therapeutics, vol. 12, no. 4, pp. 425-431, 1996.

[26] A. W. Thomson, C. A. Bonham, and A. Zeevi, "Mode of action of tacrolimus (FK506): molecular and cellular mechanisms," Therapeutic Drug Monitoring, vol. 17, no. 6, pp. 584-591, 1995.

[27] US Food and Drug Administration, "Public health advisory: Elidel (pimecrolimus) Cream and Protopic (tacrolimus) Ointment," US Food and Drug Administration, Rockville, Md, USA, 2005, http://www.fda.gov/Drugs/DrugSafety/PostmarketDrugSafetyInformationforPatientsandProviders/DrugSafetyInformationforHeathcareProfessionals/PublicHealthAdvisories/UCM051760.

[28] S. L. Schreiber and G. R. Crabtree, "The mechanism of action of cyclosporin A and FK506," Immunology Today, vol. 13, no. 4, pp. 136-142, 1992. 

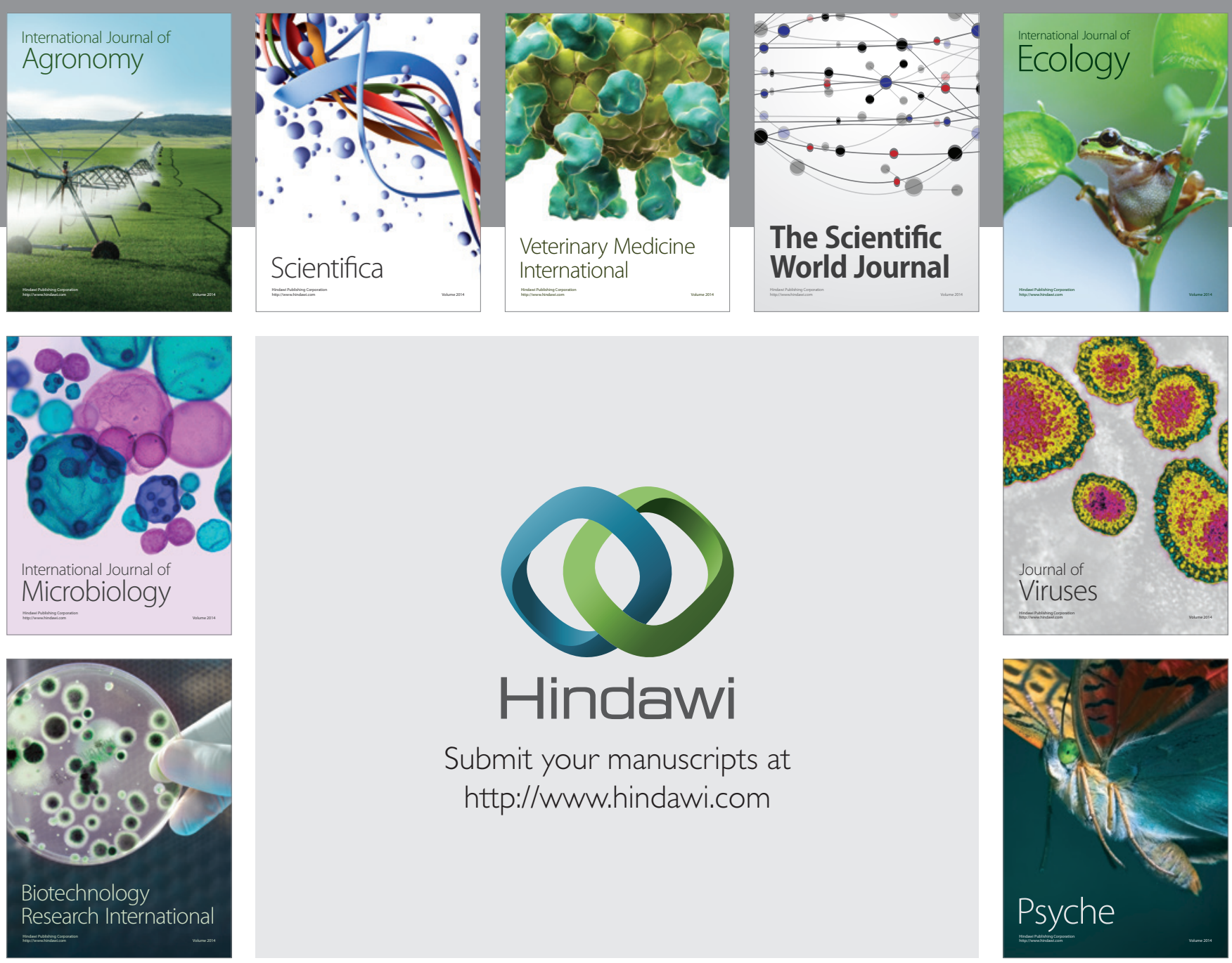

Submit your manuscripts at

http://www.hindawi.com
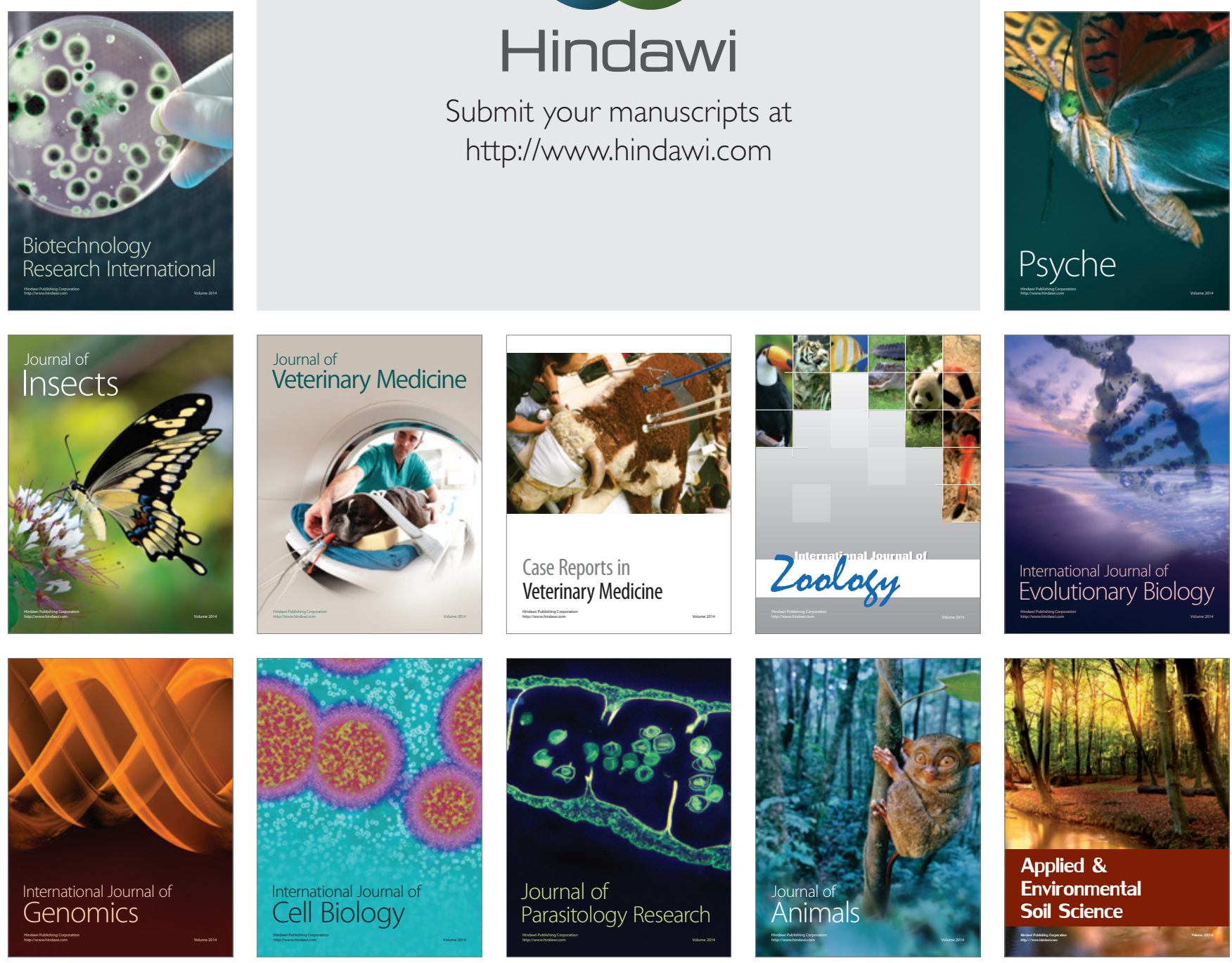